\title{
Study on Testing Technology and Application of Road and Bridge Test
}

\section{Gang Wang}

\author{
The Engineering \& Technical College of Chengdu University of Technology, Leshan, Sichuan, \\ 614000
}

\section{Keywords: Road and Bridge Test, Application Technology, Development Aspects}

\begin{abstract}
With the rapid development of Chinese economy, the scale of road and bridge engineering is getting bigger and bigger, and its construction environment is very complicated. Therefore, in the construction of road and bridge engineering, we must take road and bridge testing, so as to ensure the quality of road and bridge engineering. This paper analyzes the necessity of carrying out the test and test in the road and bridge engineering, and then focuses on the roadbed test detection technology and its application, which aims to provide reference for the construction of the road and bridge engineering.
\end{abstract}

\section{Introduction}

In the construction of road and bridge, engineering materials inevitably exist in a variety of defects, and engineering structure, construction and construction errors are inevitable, so after the completion of road and bridge construction, how to determine the quality and quality of road and bridge to become the core issue. It is well known that after the production of cars and ships, the performance and quality are tested by destructive tests, but the road and bridge is a single piece and can not carry out such destructive tests. Therefore, the detection of road and bridge quality testing technology has become the focus of attention. Tour inspection is relatively simple, but the shortcomings are obvious, and the test results are very different. Because there is no road bridge detection of quantitative methods, resulting in some of the project can not be found and dealt with rubbish. The importance of road and bridge test is mainly manifested in the following aspects: First, through the road and bridge test, you can detect the use of materials used in road and bridge qualified to determine the choice of what materials to see if the local materials; Second, through the road and bridge test, can effectively promote the new technology, new materials and new technology in the construction of road and bridge construction, and these new technologies, new materials and new technology applied to the bridge construction, can improve the quality of bridge construction, Construction and accumulated experience, to promote the rapid development of road and bridge construction; Third, the road and bridge test detection, mainly the use of scientific methods, the construction of the road and bridge a variety of materials and semi-finished products to be tested by testing to determine these materials and semi-finished products Fourth, the road and bridge testing can objectively evaluate the quality of road and bridge, and the quality of the project have a unified quality standards, road and bridge testing will help the development of a unified standard of road and bridge quality. The importance of road and bridge test is mainly manifested in the following aspects: First, through the road and bridge test, you can detect the use of materials used in road and bridge qualified to determine the choice of what materials to see if the local materials; Second, through the road and bridge test and can effectively promote the new technology, new materials and new technology in the construction of road and bridge construction, and these new technologies, new materials and new technology applied to the bridge construction, can improve the quality of bridge construction, Construction and accumulated experience, to promote the rapid development of road and bridge construction; Third, the road and bridge test detection, mainly the use of scientific methods, the construction of the road and bridge a variety of materials and semi-finished products to be tested by testing to determine these materials and semi-finished products Fourth, the road and bridge testing can objectively evaluate the quality of road and bridge, and the quality of the project have a unified quality standards, road and bridge testing will help the development of a unified standard of road and bridge quality. 


\section{Bridge Test Content and the Existence of the Problem}

Road and bridge test detection content is more extensive, and the main test content, including surface damage, the surface curve. The distribution of cracks, the width of cracks, the strength of concrete, stress and vibration, etc., including the bridge surface, stress and winding, as well as stiffness and strength of three aspects. At present, for different test items, the test method used is also different, and in the process of testing the road and bridge surface, the use is still the traditional method of visual inspection, this test method, the accuracy of the results due to People, and monitoring staff of professional quality and professional quality have a great relationship, and because it is manual detection, subjective strong, lack of scientific and rational. As we all know, the construction of road and bridge construction under normal circumstances to the main concrete structure, so the detection of concrete cracks in the road and bridge testing and testing of important content, but also a key test items. For the concrete structure of the road and bridge, the crack situation to detect and evaluate the relevant data show that: road and bridge damage is the main reason for the emergence of cracks in road and bridge. In addition, the concrete peeling, the collapse of the bridge and the bridge jump is also the reason for the cracks in concrete. Therefore, in the road and bridge test detection and should be found in the structure of the various cracks, analysis of the causes and hazards of the extent of the development of scientific methods to provide a basis for repair.

At present, the basic parameters of the road and bridge foundation in China are the numerical values of the elastic modulus EO. Although these regulations stipulate the strength of the foundation material of the road and bridge foundation, the construction process of the road and bridge construction is usually based on the specific Engineering compaction degree to strictly control the compaction degree of the ground, and this phenomenon is the most direct consequence of the design indicators and construction of construction indicators are not unified, leading to the design of the design standards lower than the design standards. However, in the test of road and bridge testing, due to the compaction degree and the elastic modulus between the functional relationship between the dynamic relationship between the control of the road and bridge foundation compaction, but it is difficult with the actual construction results anastomosis test detection of the most fundamental purpose is to objectively reflect the quality of road and bridge, through the relevant indicators to reflect the quality of road and bridge data. However, the current test indicators and usually can not accurately reflect the quality of road and bridge, and this is mainly due to errors in the test. The main reason for this situation is that the detection equipment is not complete, so it caused the road and bridge test results error.

One of the problems in the road and bridge test detection is that the test object can not objectively reflect the true quality of the road and bridge. The main reason is that the detection equipment is not perfect. Road and bridge test detection of more content, the use of test equipment is also more, whether it is from the type of speaking, or from the number of speaking, coupled with the detection is a comprehensive test, it will inevitably be operational errors, or confused Various detection equipment.

\section{Road and Bridge Test Detection Technology and Application}

At present, Chinese road and bridge surface to take the degree of compaction test point of view, most of the traditional approach, mainly sanding method "ring knife" embedded accelerometer method "nuclear emission method" static pressure method. In the current situation, in the test to check the surface of the bridge surface compaction degree, the main approach is to take a static sampling test, in such conditions, it is generally difficult to get a more realistic test results. In all the road and bridge compaction degree of detection method, ring knife method and sand filling method of the two detection methods of the results obtained is relatively accurate. However, these two detection methods also have their own shortcomings, mainly in two aspects: First, the measurement takes a long time; Second, with these two methods for testing, the need for road and bridge surface related Processing, to a certain extent, will destroy the existing road and bridge surface. Therefore, 
in the use of these two methods, we must pay attention to the damaged road and bridge surface repair. In the road and bridge test detection using embedded accelerometer method, the general will take the embedded measures, and then the road and bridge surface of the relevant compaction test, the effect is better. However, this method itself has a defect, because this method is occurred in the construction of the road and bridge, etc. After the completion of the embedded, it is not convenient to remove it, so this method can only be used once.

This method is also known as the detection of radar technology, it has an intuitive "high-precision" fast and many other advantages of the economy, this technology is generally the first use of the role of geological radar, constantly to the object to launch high-frequency electromagnetic waves, and then through the geological radar Receiving the role, from the object to obtain a variety of corresponding reflection information, and finally to further determine the various information within the object. The test of this technology has its own set of processes, generally divided into the following steps: First, by the relevant detectors through the notebook on the control unit to issue command information; Second, the control unit and then the antenna information related operations, Including the transmission of the antenna and the antenna to receive two aspects of the signal; and then "the control unit to send the signal will trigger the signal, the electromagnetic wave will be related to the information reflected to the ground, the transmission to the notebook; Finally, the detection staff through information processing technology, The internal situation of the inspection object.

This technology is generally through the heavy hammer free fall, the road and bridge surface to produce a certain impact, in the process of measuring the bridge surface deflection. This detection technique has a large difference from other detection techniques: it can be simulated in the case of vehicle travel, and then the road and bridge related to the situation, this detection method because of their own unique, so generally meet the specific traffic conditions. The use of this method in the test of the bridge surface of the deflection, but also has two advantages: First, to further improve the detection rate; Second, to a certain extent, improve the results of road and bridge testing, so by road and bridge test personnel Favored. In addition, this detection technology uses the instrument for the laser, which can detect the settlement rate of the road and bridge.

The basic principle of this detection technology is in the detection process, the first full use of ultrasonic detectors and acoustic transducer related functions, the ultrasonic pulse in the road bridge shown in the various parameters of the measurement and analysis, where the Parameters generally include the propagation speed "amplitude" of the main frequency, etc., the relevant detectors and then through these parameters and the corresponding changes as the basis for the analysis of the possible defects in the road bridge to judge. This kind of detection technology can penetrate the concrete structure, but also in the concrete to carry out, has two advantages, that is easy to operate "In general, the most commonly used ultrasonic detection method is the penetration test However, this technique is due to its own equipment limitations, so there is a drawback, that is, in the detection process requires the use of two relative test surface. In addition, because people can not determine whether the ultrasonic test, we effectively capture the defect signals of road and bridge, so to a certain extent affected the development of the technology. Thus, in the road and bridge experimental testing, in order to make up for this problem, generally take more data points, first all data And then use the probability of statistical principles to deal with the detection of the data obtained, combined with the information obtained after the assessment of road and bridge defects. Therefore, with ultrasonic testing technology test road and bridge, its intuitive poor, need to take more Point detection method to improve the detection accuracy.

\section{Conclusion}

With the speed of Chinese new urbanization, Chinese highway and bridge construction has never been developed. At the same time, the number of road and bridge construction has increased significantly. In this case, the discussion of road and bridge detection technology and its application will have important significance. 


\section{References}

[1] Li Chengyi. Effective measures to strengthen the test and test technology of expressway [J]. Heilongjiang Transportation Science and Technology, 2015 (02)

[2] Chen Xiaolin. Highway engineering test detection problems and measures [J]. Value Engineering, 2016 (30)

[3] Qian Jianhua. Highway safety detection technology [J]. Technology and Innovation, 2014 (23)

[4] Fu Sheng. Talking about the design and testing method of road and bridge [J]. Electronic production, 2014 (23)

[5] Wang Jun. Exploring highway bridge detection technology [J]. Sichuan Cement, 2015 (03)

[6] Zhang industry. Detection technology in the application of road and bridge construction analysis [J]. Chinese new technology and new products, 2016 (07) 